\title{
Real-World Treatment Patterns and Overall Survival of Patients with Metastatic Castration-Resistant Prostate Cancer in the US Prior to PARP Inhibitors
}

\author{
Neal D. Shore · François Laliberté - Raluca Ionescu-Ittu (D) • \\ Lingfeng Yang · Malena Mahendran · Dominique Lejeune • \\ Louise H. Yu • Joseph Burgents · Mei Sheng Duh · Sameer R. Ghate
}

Received: March 18, 2021 / Accepted: June 8, 2021 / Published online: July 19, 2021

(C) The Author(s) 2021

\begin{abstract}
Introduction: Therapeutic options for metastatic castration-resistant prostate cancer (mCRPC) patients are continuously advancing. We described mCRPC treatment patterns in the US from 2013 to 2019.

Methods: Patients with a confirmed mCRPC diagnosis and adenocarcinoma histology were included in the US Flatiron Health Electronic Health Record-derived de-identified database. Treatment patterns [including treatment per lines of therapies (LOTs), LOT sequences, and
\end{abstract}

Supplementary Information The online version contains supplementary material available at https:// doi.org/10.1007/s12325-021-01823-6.

N. D. Shore

Carolina Urologic Research Center, 823 82nd Pkwy

Suite b, Myrtle Beach, SC 29572, USA

F. Laliberté · R. Ionescu-Ittu (ه) · M. Mahendran .

D. Lejeune

Analysis Group, Inc., 1190 Avenue des Canadiensde-Montréal, Suite 1500, Montréal, QC H3B 0G7,

Canada

e-mail: Raluca.Ionescu-Ittu@analysisgroup.com

L. Yang · J. Burgents · S. R. Ghate

Merck \& Co., Inc., 2000 Galloping Hill Rd,

Kenilworth, NJ 07033, USA

L. H. Yu · M. S. Duh

Analysis Group, Inc, 111 Huntington Ave 14th

Floor, Boston, MA 02199, USA time on treatment] and overall survival (OS) have been described in mCRPC settings.

Results: Of 5213 patients (mean age: 72.6 years), $4374(83.9 \%)$ were treated with $\geq 1$ LOT post-mCRPC diagnosis (among those with $\geq 1$ LOT, $55.3 \%, 29.5 \%, 14.7 \%$, and $6.7 \%$ had $\geq 2,3,4$, and 5 LOTs, respectively). In first line $(1 \mathrm{~L})$, the main treatment class was nextgeneration hormonal agents (NHA; $62.5 \%$ of patients with $\geq 1$ LOT), while the shortest and longest time on $1 \mathrm{~L}$ were observed for chemotherapy (median 2.8 months) and NHA (median 5.1 months), respectively. The most common LOT sequences were NHA $\rightarrow$ NHA $(29.4 \%$ of patients with $\geq 2$ LOTs) and $\mathrm{NHA} \rightarrow \mathrm{NHA} \rightarrow$ chemotherapy $\quad(16.7 \% \quad$ of patients with $\geq 3$ LOTs). In Kaplan-Meier analyses, the median OS was 19.4, 14.6, and 11.1 months post-1L, 2L, and 3L start, respectively. Patients who moved rapidly through LOTs had an increased risk of death.

Conclusions: NHA were widely used as 1L therapy in mCRPC patients from 2013 to 2019, but time on 1L NHA treatment was on average $<6$ months. While NHA $\rightarrow$ NHA was the most observed $1 \mathrm{~L} \rightarrow 2 \mathrm{~L}$ LOT sequence, a plethora of other LOT sequences were observed. OS was poor, highlighting an unmet need for lifeprolonging treatments.

Keywords: Chemotherapy; Electronic medical records; Lines of therapy; Next-generation hormonal agents; Treatment duration 


\section{Key Summary Points}

Why carry out this study?

Metastatic castration-resistant prostate cancer (mCRPC) represents the most advanced form of prostate cancer and is associated with a poor prognosis.

Although therapeutic options for mCRPC are rapidly advancing, there is limited real-world data on contemporary treatment patterns to benchmark the changes and inform clinical practice.

To identify current gaps in the therapeutic landscape, the present retrospective study used a large oncology electronic medical records database from the US to describe treatment patterns among patients with mCRPC during the study period spanning from 2013 to $2019(n=5213)$.

\section{What was learned from this study?}

Post-mCRPC diagnosis, consecutive lines of therapy (LOT) with next-generation hormonal agents (NHA; e.g., abiraterone and enzalutamide) were the preferred treatment option in first and second line (1L and $2 \mathrm{~L} ; 29.4 \%$ of patients with $\geq 2$ LOTs), followed by consecutive lines with NHA and chemotherapy ( $16.5 \%$ of patients with $\geq 3$ LOTs); beyond third line (3L), there was an increasing dependence on chemotherapy.

Treatment duration was short across all LOTs (median: $<6$ months per LOT) and median overall survival (OS) became shorter with each successive LOT (median OS estimated in Kaplan-Meier analyses: $19.4,14.6$, and 11.1 months post-1L, $2 \mathrm{~L}$, and $3 \mathrm{~L}$ start, respectively); approximately $50 \%$ of patients in the study did not receive a subsequent LOT after $1 \mathrm{~L}$.

The study results highlight the aggressive nature of $\mathrm{mCRPC}$ and the unmet need for life-prolonging treatments in this population of patients.

\section{DIGITAL FEATURES}

This article is published with digital features, including a summary slide, to facilitate understanding of the article. To view digital features for this article go to https://doi.org/10.6084/ m9.figshare.14744073.

\section{INTRODUCTION}

Prostate cancer (PC) is one of the most commonly diagnosed cancers and a leading cause of death among men in the United States (US) $[1,2]$. Within 5 years of the PC diagnosis, an estimated $10-20 \%$ of patients develop castration-resistant prostate cancer (CRPC) [3, 4]. Metastatic CRPC (mCRPC) represents the most aggressive form of PC disease and is associated with a poor prognosis [5]. Further, mCRPC is a molecularly heterogeneous disease, with approximately $20-30 \%$ of patients harboring defects in DNA repair genes, including homologous recombination repair (HRR) gene alterations [6].

Despite the availability of next-generation hormonal agents (NHA; e.g., abiraterone and enzalutamide) for the treatment of $\mathrm{MCRPC}$, as well as other therapies [e.g., sipuleucel-T (Sip-T) immuno-oncology, radium-223, cabazitaxel] [7], treatment response is short-lived due to tumor resistance [8], and patient median overall survival (OS) remains $\leq 2$ years [9-11]. However, the therapeutic landscape for mCRPC is poised to change with the 2020 approval of polyadenosine diphosphate-ribose polymerase inhibitors (PARPi), including olaparib and rucaparib, for patients harboring HRR gene alterations who have progressed on prior treatments [12, 13].

In the Phase 3 PROfound trial [12], 387 patients with mCRPC who progressed on NHA and had alterations in at least 1 of 15 HRR genes (e.g., BRCA1/2, ATM, CDK12, FANCA, PALB2) were randomized to either olaparib or a physician's choice of NHA (i.e., abiraterone or enzalutamide). Among patients with alterations in $B R C A 1 / 2$ or $A T M$, olaparib improved the primary endpoint of imaging-based progression- 
free survival (PFS) compared to physician's choice of NHA (median PFS: 7.4 vs. 3.6 months) [12]. In the single arm TRITON2 clinical trial ( $n=209$ patients with HRR-mutated mCRPC) $[14,15]$, an objective response rate of $44 \%$ was obtained among 115 patients with BRCA1/2mutated mCRPC treated with rucaparib after progression on androgen receptor-directed therapy and taxane chemotherapy.

Based on the results of these two trials, olaparib and rucaparib were the first PARPi to be approved by the US Food and Drug Administration (FDA) in May 2020: olaparib was approved for use in mCRPC patients with at least 1 of 14 HRR gene alterations, including $B R C A 1 / 2$ alterations, who progressed on prior therapy with abiraterone or enzalutamide; rucaparib was approved for use in mCRPC patients with $B R C A 1 / 2$ alterations who have progressed on prior NHA and taxane chemotherapy. Both agents were also included in the v2.2020 NCCN Clinical Practice Guidelines In Oncology (NCCN Guidelines ${ }^{\circledR}$ ): olaparib as a Category 1 recommended therapy post-abiraterone or enzalutamide for mCRPC patients with alterations in 14 HRR genes, and rucaparib as a Category $2 \mathrm{~A}$ recommended therapy for mCRPC patients with $B R C A 1 / 2$ alterations post-androgen receptor-directed therapy and taxane-based therapy [16].

While PARPi are expected to change the treatment landscape among eligible mCRPC patients harboring HRR gene alterations, there is limited real-world data on the treatment patterns of these patients in the pre-PARPi era to benchmark the changes. Although real-world treatment patterns after MCRPC diagnosis have been evaluated in a few recent studies in the US $[10,11,17-19]$, multiple knowledge gaps remain $[10,11]$. First, line of therapy (LOT) sequences described in these prior studies do not go beyond the third line $(3 \mathrm{~L})$ in the mCRPC setting. Second, treatment patterns were described at agent level only [10, 11], while analyses at treatment class level could provide a broader perspective, particularly as novel PARPi are available to patients who have responded inadequately to specific classes of treatments (e.g., NHA). Third, information regarding the duration of treatments is lacking for specific
LOT sequences, including the time on and off treatments. Finally, there is a need for updated real-world OS estimates in this population, including the impact of the number of LOTs on patients' OS. The present study provides a contemporary account of real-world treatment patterns among mCRPC patients in the US that addresses these limitations, with the aim of identifying current treatment gaps that newly emerging therapies could fill. The study focuses on treatment patterns among all patients with mCRPC (given that no targeted treatment options were approved for patients harboring HRR gene alterations during the 2013-2019 study period, the treatment patterns in the overall mCRPC population will serve as proxy for the treatment patterns of patients with mCRPC harboring HRR gene alterations in the pre-PARPi era).

\section{PATIENTS AND METHODS}

\section{Data Source}

Adults diagnosed with mCRPC were included from January 1, 2013 to March 31, 2019 based on the US Flatiron Health Electronic Health Record (EHR)-derived de-identified database, an oncology EHR database that has been previously used to investigate treatment patterns and outcomes in mCRPC $[10,11,20]$. The US Flatiron Health database is a longitudinal database, comprising de-identified patient-level structured and unstructured data, curated via technology-enabled abstraction [21]. Patients in Flatiron data were shown to be similar in sex and geographical distribution to patients in US cancer registries, such as the Surveillance, Epidemiology, and End Results Program or the National Program of Cancer Registries, but appeared to be diagnosed with later stages of disease and had different age distribution compared to patients in the cancer registries [22].

The Flatiron Health EHR-derived de-identified database [21] includes normalized data elements (e.g., demographics, diagnoses, visits, laboratories/vitals results, medication administration/orders/prescriptions, performance status), insurance), enhanced data elements 
curated specifically for the mCRPC target population (e.g., date of the initial PC diagnosis, cancer stage at the initial PC diagnosis Gleason score at the initial PC diagnosis, date of first metastasis, date of CRPC diagnosis, and LOTs), and month/year of death.

All database records were statistically deidentified and certified as fully compliant with US patient confidentiality requirements outlined in the Health Insurance Portability and Accountability Act of 1996. Since this study relied exclusively on de-identified patient records and did not involve the collection, use, or dissemination of individually-identifiable data, institutional review board approval was not necessary.

\section{Study Design and Population}

A retrospective longitudinal observational cohort design was used (Supplemental Fig. 1). The study sample included males aged 18 years and older with mPC and CRPC diagnoses between January 1, 2013 and March 31, 2019 and adenocarcinoma histology (Supplemental Fig. 2). The date of mCRPC diagnosis was defined as either the date of MPC or the date of CRPC diagnosis, whichever came later. If the mPC date preceded the CRPC date, the patient was assumed to have transitioned to MCRPC from the metastatic hormone-sensitive PC clinical state (mHSPC); if the CRPC date preceded the MPC date, the patient was assumed to have transitioned to MCRPC from the nonmetastatic CRPC clinical state (nmCRPC).

Baseline characteristics were measured from the initial PC diagnosis to the MCRPC diagnosis date, as recorded in the EHR data. Patients were followed from the mCRPC diagnosis date to the end of observed clinical activity in Flatiron data or death. All patients had at least 1 month of follow-up post-index by design (Supplemental Figs. 1, 2). No minimum number of documented treatments was required at study entry.

\section{Outcomes and Measurements}

Patient demographic and clinical characteristics were measured during the baseline period and at the index date, based on information available in the normalized and enhanced Flatiron Health EHR-derived de-identified data. The proportion of patients treated at oncology centers in community settings versus academic centers was reported.

Treatment patterns pre-mCRPC diagnosis included the different classes of treatments used between the PC diagnosis and MCRPC diagnosis, both overall and during relevant premCRPC clinical states [i.e., non-metastatic hormone-sensitive PC (nmHSPC), mHSPC, and nmCRPC clinical states]. A clinical states model has been previously used to track the progression of disease from the initial PC diagnosis to mCRPC diagnosis and to identify patient populations most in need of treatment [23-25].

Treatment patterns post-mCRPC diagnosis included the number of observed LOTs per patient, LOT sequences post-mCRPC diagnosis by treatment class (identified using a PC-specific proprietary algorithm [21]), regimens by LOT (class and agent-level), time between the date of mCRPC diagnosis and first line (1L) start, and time on and off treatment for LOTs by class. The number of observed LOTs per patient were evaluated both overall and among the subgroup of patients who died during the study period. For the latter, the number of observed LOTs per patient was not affected by censoring due to end of data availability, and thus provides a more accurate picture of the total number of LOTs the patient received before succumbing to the disease.

For the regimens by LOT analyses, the following five treatment groups were analyzed at class level: NHA (i.e., abiraterone, enzalutamide, apalutamide, and darolutamide), chemotherapy (e.g., docetaxel, cabazitaxel), Sip-T, and other therapy (i.e., thalidomide, bcg vaccine, nivolumab, atezolizumab, lenolidomide, durvalumab, ipilimumab, pembrolizumab, targeted therapies, radium-223, and clinical study drugs; nonNHA hormonal therapies or bone therapy agents were not included in concordance with the LOT algorithm), as well as any combinations of the four classes above.

For all classes of therapy, time on a given LOT was defined as the time from the first order/administration to the last order/ 
administration of the agents in the LOT (for chemotherapy, this definition is conservative, as the full cycle of the last administration was not included), irrespective of censoring. Similarly, time off treatment was defined as the time between the last order/administration for a given LOT and the first order/administration for the next LOT. Given that the last LOT observed may be censored due to the end of data availability and thus underestimated, the proportion of patients with last LOT censored is also reported. When the last LOT was an IV therapy, censoring was defined as end of data availability due to end of clinical activity or death within 62 days of the last administration of the IV therapy; when the last LOT was an oral therapy, censoring was defined as end of data availability within 90 days of the last day supply of the oral therapy.

Finally, OS was assessed by LOT, after the start of $1 \mathrm{~L}, 2 \mathrm{~L}$, and $3 \mathrm{~L}$, respectively, accounting for censoring. Further, we also assessed whether patients who move rapidly through multiple LOTs have worse OS than patients who advance slower through multiple LOTs.

\section{Statistical Analysis}

Baseline characteristics and treatment patterns were analyzed using descriptive statistics [i.e., proportions, means, standard deviations (SD), medians, and interquartile ranges (IQR)]. The median time on and off treatment (months) and the corresponding IQRs were assessed across patients' LOT sequences based on the patients' observed time on/off treatment during the study period, irrespective of censoring.

OS was analyzed using time to event analyses among patients who did not have other primary malignancies occurring concurrently with mCRPC, and who did not participate in randomized control trials (RCT). This analysis relied on a composite mortality variable described previously [26]. Kaplan-Meier (KM) analyses were used to assess OS after $1 \mathrm{~L}, 2 \mathrm{~L}$, and $3 \mathrm{~L}$ start and to derive median OS and OS rates. Patients without a death event after each LOT start were censored at the end of observed clinical activity in Flatiron Health EHR-derived de-identified data. In addition, we reported results from a multivariate Cox proportionalhazards regression model that assessed the impact of LOT number post-mCRPC diagnosis (i.e., $1 \mathrm{~L}, 2 \mathrm{~L}, 3 \mathrm{~L}$ or $4 \mathrm{~L}+$; modeled as time-dependent exposure) adjusted for the following covariates measured at or before mCRPC diagnosis (modeled as fixed or time-independent covariates): age, time from initial PC diagnosis to mCRPC diagnosis, ECOG performance status, disease progression to MCRPC, Gleason score, hemoglobin level, lactose dehydrogenase level, albumin level, serum alkaline phosphatase level, prostate-specific antigen (PSA) level, site of metastasis, opioid use, cancer stage, prostatectomy/surgery, and radiation. The hazard ratios for this time-dependent exposure compared the risk of death between patients who had more versus fewer LOTs at each point in time post-mCRPC diagnosis, adjusting for potential confounders. Univariate Cox regression models were also performed for both the main exposure and each covariate.

All analyses were performed using SAS Enterprise Guide v.7.1 (SAS Institute, Cary, NC, USA).

\section{RESULTS}

\section{Baseline Characteristics}

Of 6883 patients with a mCRPC diagnosis in the database between 2013 and 2019, a total of 5213 patients were included in the mCRPC study sample (Supplemental Fig. 2). The mean time from the initial PC diagnosis to the mCRPC diagnosis was 5.8 years and the mean time from the start of clinical activity to the mCRPC diagnosis was 2.2 years (Table 1 ). The mean age of the patients was 72.6 [median 74] years and the majority were White $(76.3 \%$; Table 1). Most patients were managed in oncology centers in the community setting (91.3 vs. $8.7 \%$ in academic setting), while about half were from the South (50.5\%) and had unknown cancer stage at the initial PC diagnosis $(43.9 \%$; Table 1), which is reflective of the Flatiron Health network rather than the US population overall [22]. At the time of the 
Table 1 Baseline characteristics of patients with $\mathrm{mCRPC}$

\begin{tabular}{|c|c|}
\hline Characteristics $^{\mathbf{a}}$ & $n=5213$ \\
\hline $\begin{array}{l}\text { Age at mCRPC diagnosis date, years, mean } \\
\text { [median] }\end{array}$ & $72.6[74]$ \\
\hline \multicolumn{2}{|l|}{ Race, $n(\%)$} \\
\hline Patients with known race & $4765(91.4)$ \\
\hline White & $3638(76.3)$ \\
\hline Others & $517(10.8)$ \\
\hline Black or African Americans & $511(10.7)$ \\
\hline Asian & $86(1.8)$ \\
\hline Hispanic or Latino & $13(0.3)$ \\
\hline Ethnicity: Hispanic or Latino, $n(\%)$ & $299(5.7)$ \\
\hline \multicolumn{2}{|l|}{ Year of mCRPC diagnosis date, $n(\%)$} \\
\hline 2013 & $413(7.9)$ \\
\hline 2014 & $816(15.7)$ \\
\hline 2015 & $937(18.0)$ \\
\hline 2016 & $1003(19.2)$ \\
\hline 2017 & $1070(20.5)$ \\
\hline 2018 & $847(16.2)$ \\
\hline 2019 (Q1) & $127(2.4)$ \\
\hline \multicolumn{2}{|l|}{ Patient's region of residence, $n(\%)$} \\
\hline Patients with known region & $4723(90.6)$ \\
\hline South & $2383(50.5)$ \\
\hline West & $856(18.1)$ \\
\hline Northeast & $750(15.9)$ \\
\hline Midwest & $734(15.5)$ \\
\hline \multicolumn{2}{|l|}{ Practice type, $n(\%)$} \\
\hline Community-based & $4762(91.3)$ \\
\hline Academic & $451(8.7)$ \\
\hline \multicolumn{2}{|c|}{ Time to mCRPC diagnosis date, years, mean [median] } \\
\hline From initial PC diagnosis & $5.8[3]$ \\
\hline From clinical activity start ${ }^{\mathrm{b}}$ & $2.2[2]$ \\
\hline \multicolumn{2}{|l|}{ PC characteristics at the initial PC diagnosis } \\
\hline \multicolumn{2}{|l|}{ Cancer stage, $n(\%)$} \\
\hline Patients with stage known & $2925(56.1)$ \\
\hline Stage I-III & $641(21.9)$ \\
\hline
\end{tabular}

Table 1 continued

\begin{tabular}{cl}
\hline Characteristics $^{\mathbf{a}}$ & $\boldsymbol{n}=\mathbf{5 2 1 3}$ \\
\hline Stage IV & $2284(78.1)$ \\
Gleason score, $n$ (\%) & \\
Patients with Gleason score known & $4349(83.4)$ \\
$\leq 6$ (low risk) & $412(9.5)$ \\
7 (intermediate risk) & $1068(24.6)$ \\
$\geq 8$ (high risk) & $2869(66.0)$
\end{tabular}

PC characteristics at the mCRPC diagnosis date ${ }^{c}$

ECOG performance status

$$
\begin{array}{ll}
\text { Patients with ECOG test, } n(\%) & 1930(37.0) \\
\text { ECOG score of } 0-1 & 1614(83.6) \\
\text { ECOG score } \geq 2 & 316(16.4)
\end{array}
$$

Laboratory test results, mean [median] (\% patients tested)

\begin{tabular}{lc} 
Hemoglobin level $(\mathrm{g} / \mathrm{dL})$ & $12.3[13](58.3)$ \\
Lactose dehydrogenase $(\mathrm{U} / \mathrm{L})$ & $247.0[195]$ \\
& $(12.5 \%)$ \\
PSA laboratory value $(\mathrm{ng} / \mathrm{mL})$ & $130.9[18]$ \\
& $(92.8)$ \\
Serum albumin $(\mathrm{g} / \mathrm{L})$ & $40.1[41](55.3)$ \\
Serum alkaline phosphatase $(\mathrm{U} / \mathrm{L})$ & $146.9[90]$ \\
& $(56.8)$ \\
\hline
\end{tabular}

$E C O G$ Eastern cooperative oncology group, EHR electronic health record, $m C R P C$ metastatic castration-resistant prostate cancer, $P C$ prostate cancer, $P S A$ prostate-specific antigen, $S D$ standard deviation

a Comorbid conditions are not reported in the current study because they are underreported in EHR data from oncology centers

b Clinical activity included any recorded interactions between the patient and a healthcare provider, including visits, use of therapies (e.g., oral or immuno-oncology, androgen deprivation therapy, radiopharmaceuticals, medication orders, and administrations) or laboratory tests, vital assessments, ECOG performance assessments, progression events, as well as death c Evaluated on the date closest to the MCRPC diagnosis date in the 3 months prior to or on the mCRPC diagnosis date 
initial PC diagnosis, most patients had Stage IV PC (78.1\%) and a Gleason score of $\geq 8$ (66.0\%; Table 1). At the date of mCRPC diagnosis, most patients had an ECOG performance status score of $0-1(83.6 \%)$ and mean PSA value of $130.9 \mathrm{ng} /$ mL (median: $18 \mathrm{ng} / \mathrm{mL}$; Table 1 ).

\section{Treatment Patterns Pre-mCRPC Diagnosis}

Most treatments observed between the PC diagnosis and the MCRPC diagnosis were hormonal therapies $(77.6 \%$ of patients in the study sample, including first-generation anti-androgen therapy $[63.6 \%$ of patients in the study sample], luteinizing hormone-releasing hormone (LHRH) agonists [34.4\%], and NHA [21.1\%]). Besides hormonal therapy, other treatment classes used before the mCRPC setting were other therapy $(29.4 \%$; largely accounted for by bone therapy agents), chemotherapy (12.4\%), and Sip-T (1.7\%) (Table 2).

Treatment patterns before the MCRPC setting were found to differ based on the PC clinical state (Table 2). Among patients diagnosed with mHSPC before mCRPC $(n=3784,72.6 \%)$, hormonal therapy was the most used therapy in both the nmHSPC and mHSPC clinical states (21.7\% and $63.5 \%$, respectively), while NHA were more commonly used by patients in the mHSPC clinical state (14.9 vs. $1.1 \%$ in nmHSPC clinical state). Chemotherapy and other therapy were rare $(<1.0 \%$ of patients used each agent) in the nmHSPC clinical state, but more common in the mHSPC clinical state $(14.3 \%$ and $36.7 \%$, respectively), whereas Sip-T was rare in both states $(\leq 1.4 \%)$. Among patients diagnosed with the nmCRPC before mCRPC $(n=1365$, $26.2 \%)$, hormonal therapy was the most commonly used therapy both in the nmHSPC and nmCRPC clinical states $(52.6 \%$ and $57.8 \%$, respectively), however with a different breakdown for the use of first-generation anti-androgens and NHA across the nmHSPC and nmCRPC clinical states: first-generation antiandrogens were more common in the nmHSPC clinical state than in the nmCRPC clinical state (46.7 vs. $27.7 \%$ ), while NHA were less commonly used in the nmHSPC clinical state than in the nmCRPC clinical state (4.0 vs. $34.1 \%)$. The remaining treatment classes were rare in both states, although they appeared to have slightly higher utilization in the nmCRPC clinical state (2.5-6.4 vs. $\leq 2.1 \%$ in nmHSPC). Among patients with MPC and CRPC diagnoses documented at the same time $(n=64,1.3 \%)$, hormonal therapy was the most commonly used therapy (53.1\%).

\section{Treatment Patterns Post-mCRPC Diagnosis}

\section{LOT Sequences}

Of 5213 patients, $4374(83.9 \%)$ were treated with $\geq 1$ LOT post-mCRPC diagnosis. Among those patients with $\geq 1$ LOT observed after the mCRPC diagnosis, 2419 (55.3\%) were treated with $\geq 2$ LOTs, 1292 (29.5\%) with $\geq 3$ LOTs, $645(14.7 \%)$ with $\geq 4$ LOTs, and $292(6.7 \%)$ with $\geq 5$ LOTs (Fig. 1 ). For $44.8 \%$ and $39.2 \%$ of patients with one and two LOTs only, respectively, a subsequent LOT was not observed because of censoring due to end of data availability. Furthermore, a large proportion of patients died after only one or two LOTs. Among the 2383 patients for whom death was observed during the study period, 957 (40.2\%) died after one LOT, 625 (26.2\%) died after two LOTs, $388(16.3 \%)$ died after three LOTs, 230 (9.7\%) died after four LOTs, and 183 (7.7\%) died after five LOTs.

Multiple distinct LOT sequences were observed post-mCRPC diagnosis; among patients with $\geq 2$ observed LOTs the most common $1 \mathrm{~L} \rightarrow 2 \mathrm{~L}$ sequences were NHA $\rightarrow$ NHA (29.4\%) and NHA $\rightarrow$ chemotherapy (16.5\%); among patients with $\geq 3$ LOTs, the most common sequence was NHA $\rightarrow$ NHA $\rightarrow$ chemotherapy (16.7\%; Fig. 2).

\section{Regimen by LOT}

Post-mCRPC diagnosis, NHA regimens were the most common regimens in earlier LOTs, whereas chemotherapy regimens were most common in later LOTs (Fig. 3). Specifically, at the agent level (Fig. 3), the most used regimens in $1 \mathrm{~L}$ and $2 \mathrm{~L}$ were abiraterone $(34.8 \%$ and $22.2 \%$, respectively) and enzalutamide (29.6\% and $28.9 \%$, respectively), while docetaxel 


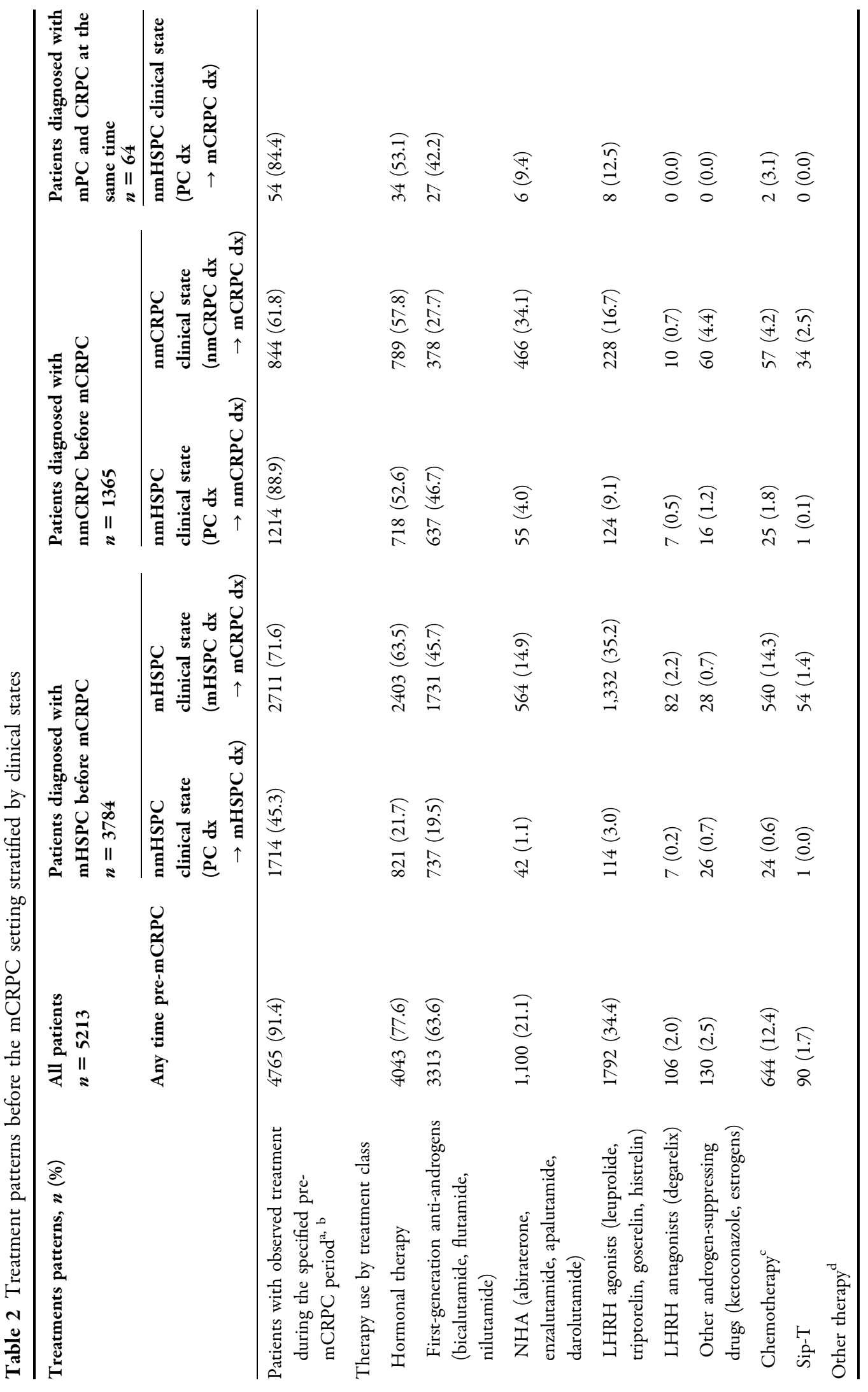




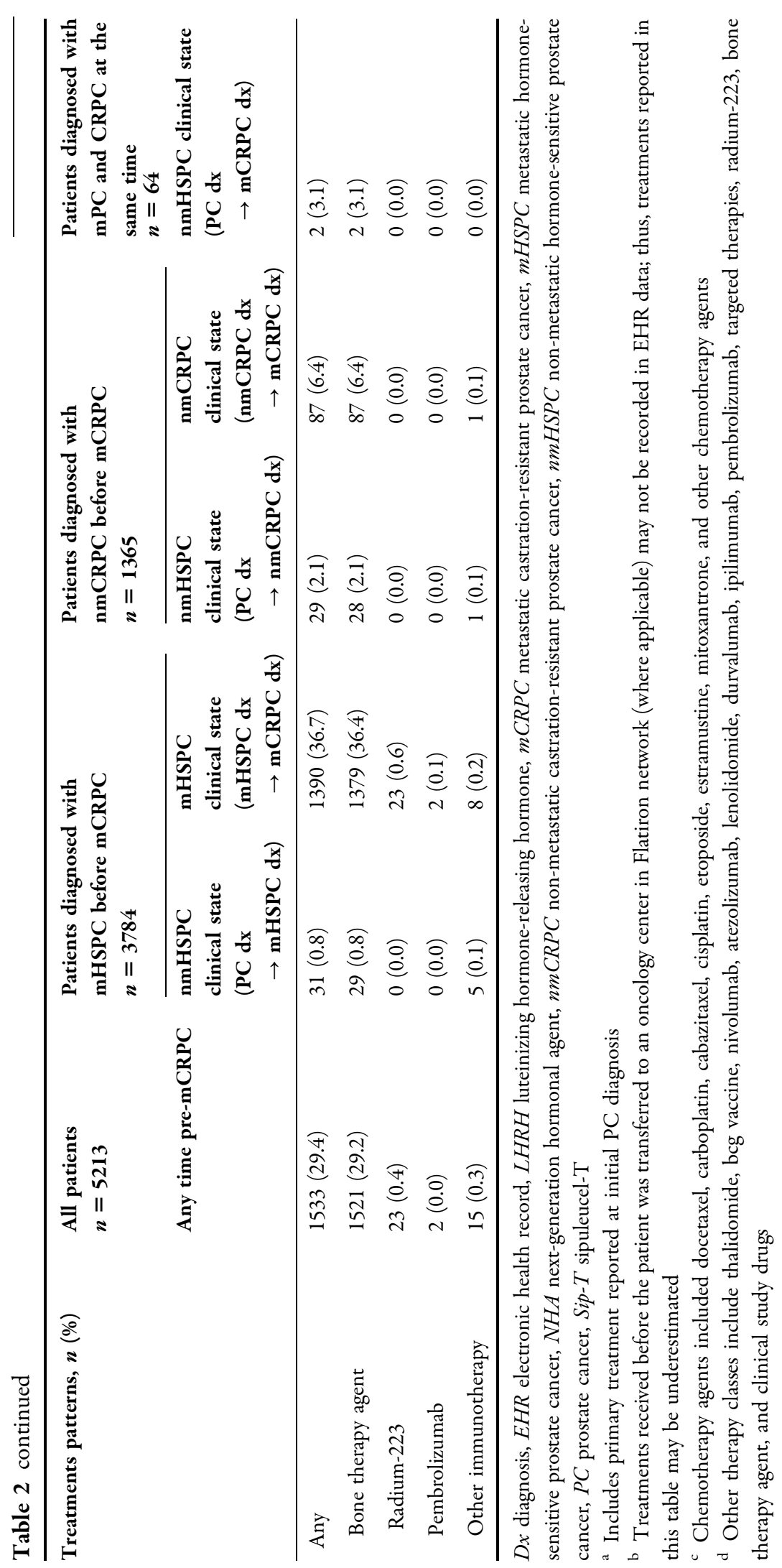




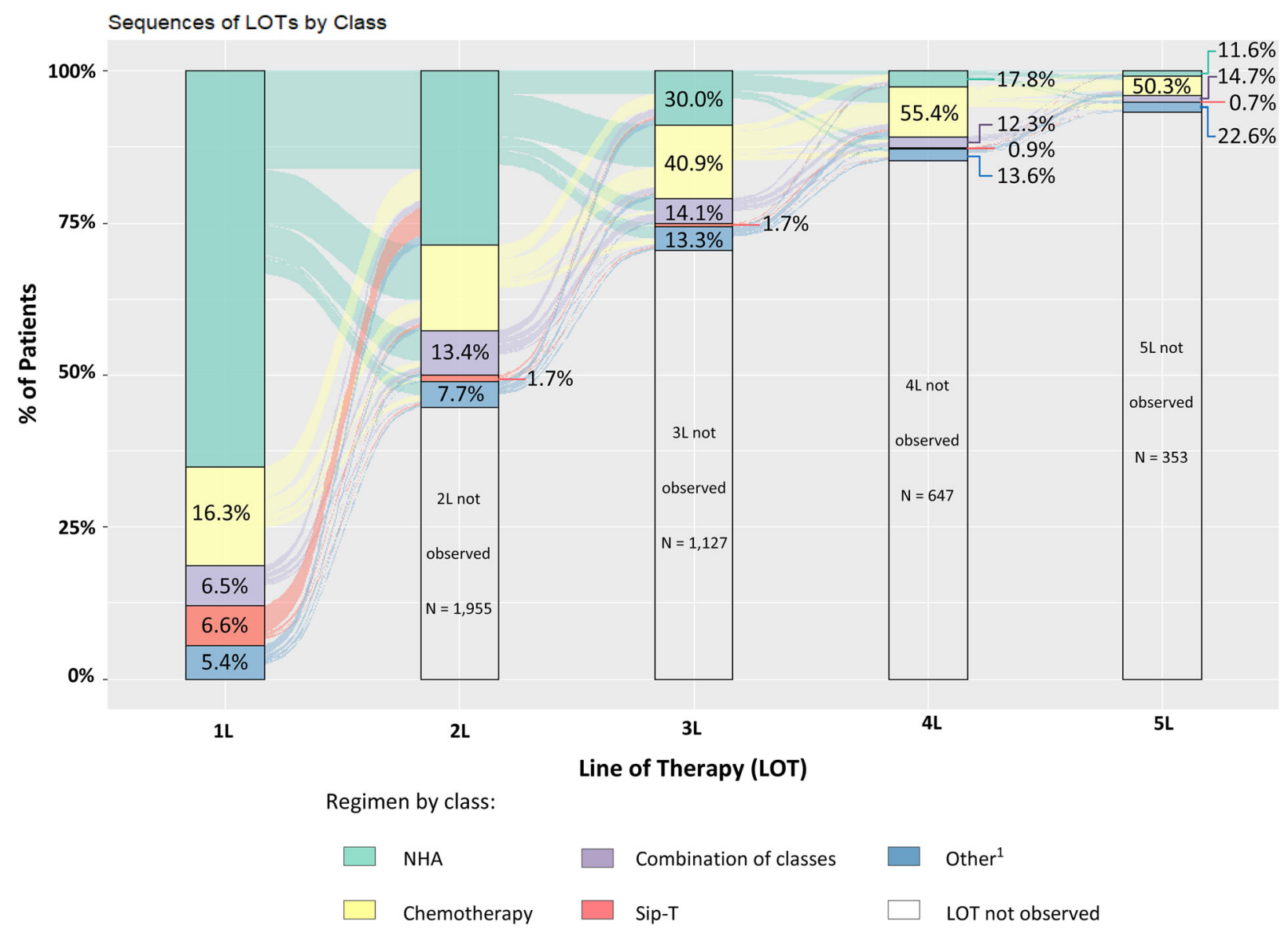

Fig. 1 MCRPC treatment patterns: number and proportion of patients by line of therapy and treatment class. $1 L$ first line, $2 L$ second line, $3 L$ third line; $4 L$ fourth line; $5 L$ fifth line; $L O T$ line of therapy, $m C R P C$ metastatic castration-resistant prostate cancer, $N H A$ next-generation

monotherapy was the most used regimen in $3 \mathrm{~L}$ $(23.2 \%)$. The most used regimens in $4 \mathrm{~L}$ and $5 \mathrm{~L}$ were cabazitaxel $(25.3 \%$ and $22.6 \%$, respectively) and docetaxel (19.2\% and $10.6 \%$, respectively).

\section{Treatment Duration by LOT Sequence and Class}

The median duration of time on and off treatment per LOT is presented in Fig. 2 for the most common LOT sequences. Among patients with $\geq 1$ LOT, the shortest and longest time on $1 \mathrm{~L}$, respectively, were observed for $1 \mathrm{~L}$ chemotherapy (median 2.8 months across all patients with $1 \mathrm{~L}$ chemotherapy) and $1 \mathrm{~L}$ NHA hormonal agent, Sip-T sipuleucel-T. 1. Other therapy classes include thalidomide, bcg vaccine, nivolumab, atezolizumab, lenolidomide, durvalumab, ipilimumab, pembrolizumab, targeted therapies, radium-223, and clinical study drugs

(median 5.1 months). Among patients with $\geq 2$ LOTs $(1 \mathrm{~L} \rightarrow 2 \mathrm{~L})$, the shortest and longest median time on $1 \mathrm{~L}$ and $2 \mathrm{~L}$ combined, respectively, were observed for chemotherapy $\rightarrow$ chemotherapy (5.2 months) and Sip$\mathrm{T} \rightarrow$ NHA (12.1 months); the longest median time off treatment was after 1L chemotherapy (1.5 months). Finally, among patients with $\geq 3$ LOTs $(1 \mathrm{~L} \rightarrow 2 \mathrm{~L} \rightarrow 3 \mathrm{~L})$, the shortest and longest median time on $1 \mathrm{~L}, 2 \mathrm{~L}$, and $3 \mathrm{~L}$ combined, respectively, were observed for NHA $\rightarrow$ chemotherapy $\rightarrow$ chemotherapy (9.1 months) and NHA $\rightarrow$ NHA $\rightarrow$ combination of classes (16.6 months); the longest median time off treatment was after $1 \mathrm{~L}$ chemotherapy 
Patients with $\geq 1$ LOT $(\mathrm{N}=4,374)$ :

$1 \mathrm{~L}$ Class

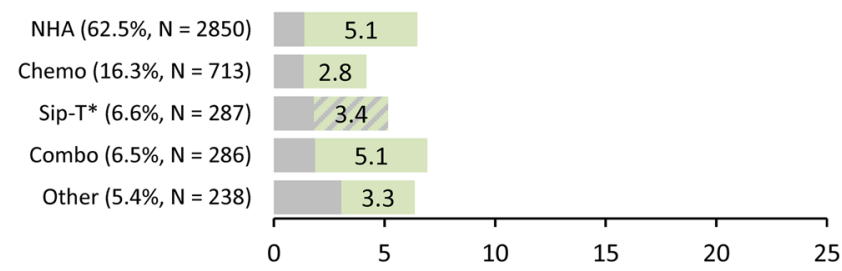

Patients with $\geq 2$ LOTs ( $N=2,419)$ :

Top $10^{1}$ Most Frequent $1 \mathrm{~L} \rightarrow \mathbf{2 L}$ Sequences by Class
mCRPC $\rightarrow$ 1L start

1L

$1 \mathrm{~L}$ end $\rightarrow 2 \mathrm{~L}$ start

2L

$2 L$ end $\rightarrow 3 L$ start

3L

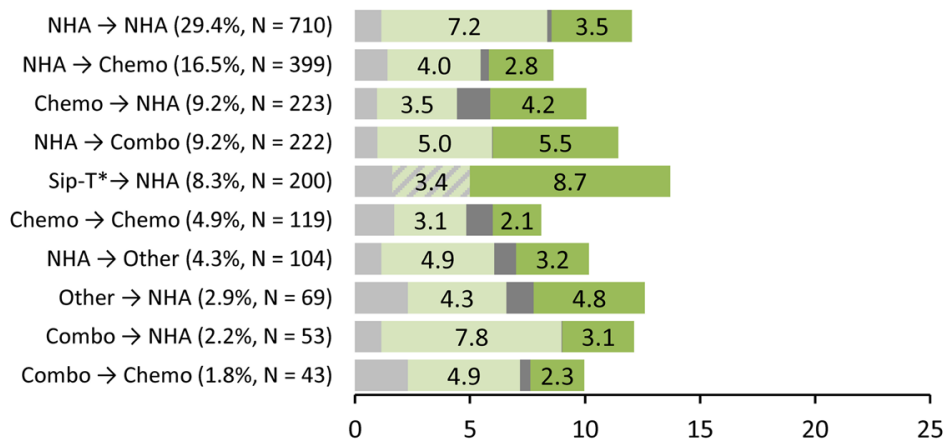

Patients with $\geq 3$ LOT ( $N=1,292)$ :

Top $10^{1}$ Most Frequent $1 \mathrm{~L} \rightarrow 2 \mathrm{~L} \rightarrow 3 \mathrm{~L}$ Sequences by Class

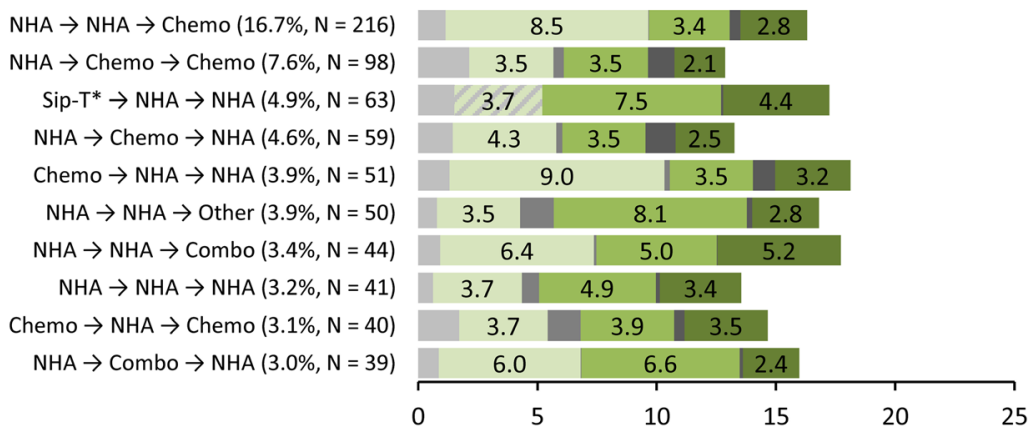

Fig. 2 mCRPC treatment sequences: time on and time off treatment. $I L$ first line, $2 L$ second line, $3 L$ third line; Chemo chemotherapy, Combo combination therapy, LOT line of therapy, $m C R P C$ metastatic castration-resistant prostate cancer, $N H A$ next-generation hormonal agent, Sip- $T$ sipuleucel-t. ${ }^{*}$ For Sip- $T$ only the start date is available in the data, and thus we cannot distinguish

(1.4 months). Although time on treatment was reported as median across all patients, some regimens/LOTs showed higher individual variability than others in time on treatment (e.g., between time on and off treatment for this agent. 1. Only the top 10 sequences are shown (of a total of 25 observed $1 \mathrm{~L} \rightarrow 2 \mathrm{~L}$ sequences and 97 observed $1 \mathrm{~L} \rightarrow 2 \mathrm{~L} \rightarrow 3 \mathrm{~L}$ sequences; of note, there are a total of 25 possible sequences for $1 \mathrm{~L} \rightarrow 2 \mathrm{~L}$ and a total of 125 possible $1 \mathrm{~L} \rightarrow 2 \mathrm{~L} \rightarrow 3 \mathrm{~L}$ sequences by combining 5 treatment classes across 2 and 3 LOTs, respectively)

IQR for time on treatment for $1 \mathrm{~L}$ NHA vs. $1 \mathrm{~L}$ chemotherapy: $2.4-10.6$ vs. 1.4-4.0 months, respectively; Supplemental Table 1). A more detailed description of median [IQR] time on 


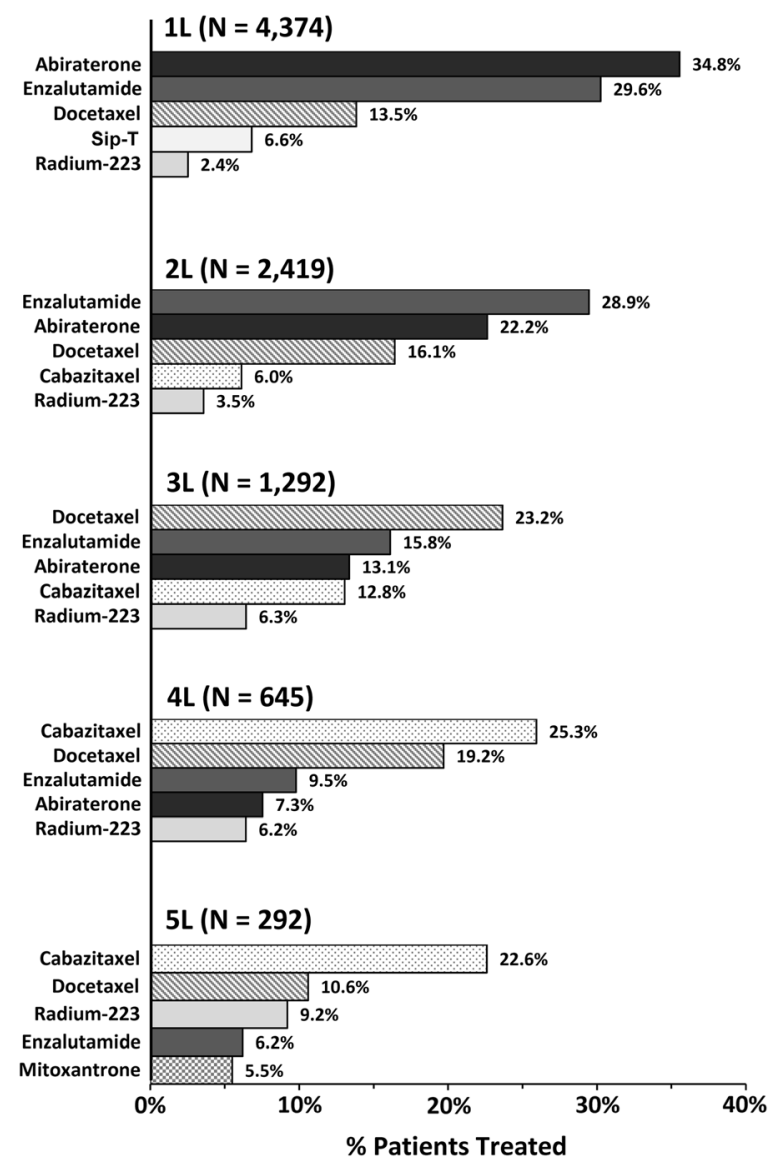

Fig. 3 Top $5^{1}$ most common regimens (agent level) by lines of therapy. $1 L$ first line, $2 L$ second line, $3 L$ third line, $4 L$ fourth line; $5 L$ fifth line; $m C R P C$ metastatic castration-resistant prostate cancer, $S i p-T$ sipuleucel-T. 1 . Top 5 regimens at an agent level out of a total of 100 regimens observed in $1 \mathrm{~L}, 123$ regimens observed in $2 \mathrm{~L}$, 94 regimens observed in 3L, 78 regimens observed in 4L, and 66 regimens observed in $5 \mathrm{~L}$

treatment is provided in Supplemental Table 1 (by class) and Supplemental Table 2 (by agent), respectively.

\section{Overall Survival}

The median OS became shorter after each new LOT start (19.4 months post-1L, 14.6 months post-2L, and 11.1 months post-3L start; Fig. 4A-C). Furthermore, the unadjusted and adjusted Cox regression models showed that the more LOTs a patient completed up to a given point in time post-mCRPC diagnosis, the higher
4 Fig. 4 Overall survival for patients with mCRPC. A Overall survival after $1 \mathrm{~L}$ start. B Overall survival after 2L start. C Overall survival after $3 \mathrm{~L}$ start. Number of patients still observed at the specific point in time: 1040 patients participating in clinical trials or having a diagnosis of another cancer were excluded from the analysis; 466 patients participating in clinical trials or having a diagnosis of another cancer were excluded from the analysis; 300 patients participating in clinical trials or having a diagnosis of another cancer were excluded from the analysis

their hazard of death compared to patients who were still on $1 \mathrm{~L}$ at that point in time (Table 3). After adjusting for potentially confounding factors, the hazard of death became progressively higher as the number of LOTs increased [HR $(95 \%$ CI) for patients with 0 LOTs: 0.86 (0.76, 0.97); 2 LOTs: 1.31 (1.17, 1.47); 3 LOTs: 1.92 (1.66, 2.23); 4 LOTs: 3.19 (2.71, 3.76); all $p<0.05]$. In the multivariate Cox regression model, other factors associated with increased hazard of death included older age, shorter time from the initial PC diagnosis to the mCRPC diagnosis, high-risk Gleason score, higher ECOG performance status, use of opioid therapy in the pre-mCRPC setting, and abnormal laboratory test results at $\mathrm{MCRPC}$ diagnosis, including higher levels of PSA (Table 3).

\section{DISCUSSION}

The findings of this real-world study indicate that consecutive LOTs with NHA were the preferred $1 \mathrm{~L}$ and $2 \mathrm{~L}$ treatment options among mCRPC patients during the 2013-2019 study period, with increasing use of cytotoxic chemotherapy beyond 2L. Approximately half of the patients had $\geq 2$ LOTs observed, and the median treatment duration was typically $<6$ months per LOT. Median OS became shorter with each successive LOT (median OS: ranged from 19.4 months from start of $1 \mathrm{~L}$ to death to 11.1 months from 3L to death), and patients who moved rapidly through multiple post-mCRPC LOTs had poorer OS compared to those who stayed longer on 1L. Taken together, these findings highlight the aggressive nature of mCRPC and the need for more effective novel 


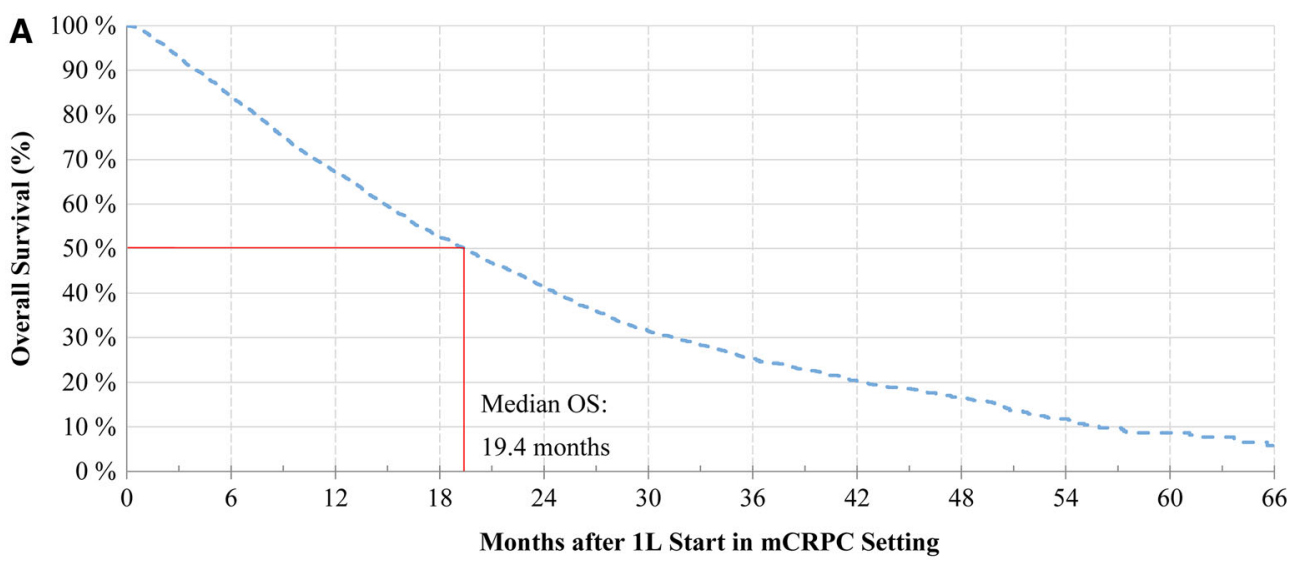
No. of patients at risk ${ }^{1}$
0 months
6 months
3,694
12 months
1,860
18 months
1,197
24 months Overall $^{2}$

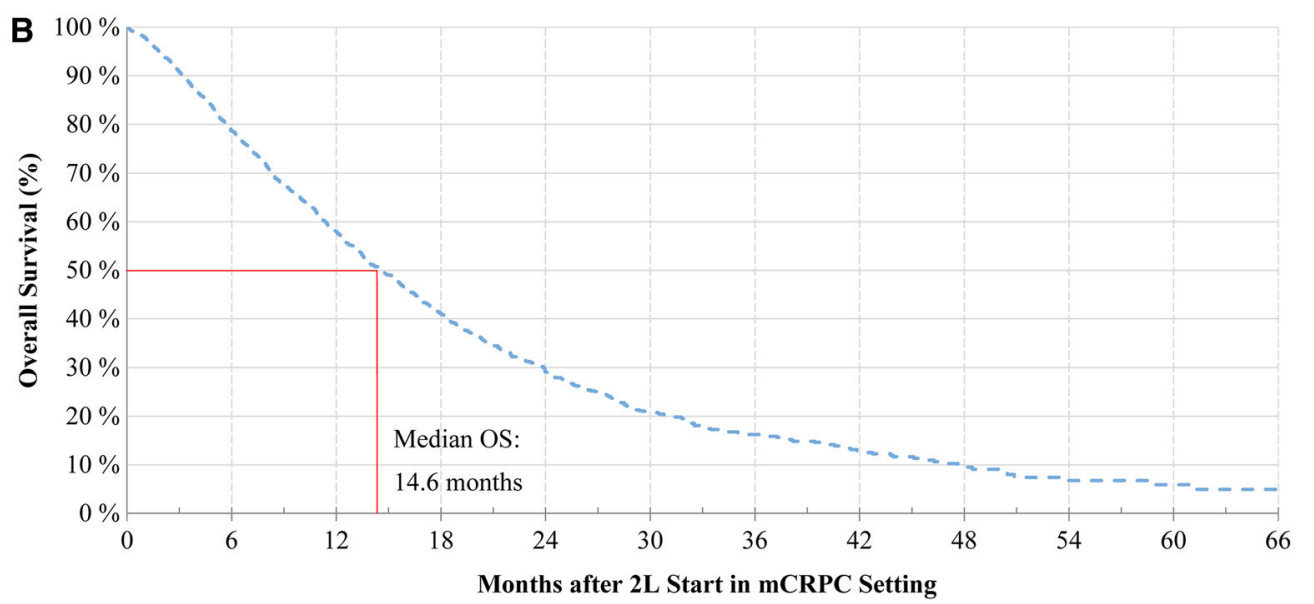
No. of patients at risk ${ }^{1}$
0 months
1,953
6 months
1,316
12 months
793
18 months
24 months Overall $^{2}$

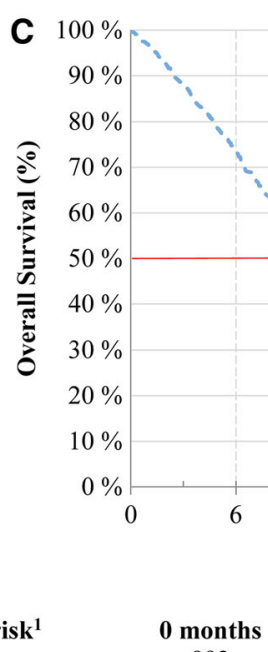

No. of patients at risk ${ }^{1}$ Overall ${ }^{2}$
6 months
589

12 months
302
18 months 167 
Table 3 Univariate and multivariate association of potential confounders and mortality among patients with mCRPC

\begin{tabular}{|c|c|c|c|c|}
\hline & \multirow{2}{*}{\multicolumn{2}{|c|}{$\begin{array}{l}\text { Univariate regression analysis } \\
\text { Overall survival (all patients, } \\
n=4436 \text { ) }\end{array}$}} & \multirow{2}{*}{\multicolumn{2}{|c|}{$\begin{array}{l}\text { Multivariate regression analysis } \\
\text { Overall survival (all patients, } \\
n=4436 \text { ) }\end{array}$}} \\
\hline & & & & \\
\hline & HR (95\% CI) & $P$ value & HR $(95 \% C I)$ & $P$ value \\
\hline \multicolumn{5}{|l|}{ LOT post-mCRPC diagnosis (time-dependent covariate) } \\
\hline \multicolumn{5}{|l|}{ LOT post-mCRPC diagnosis (ref: $1 \mathrm{~L}$ ) } \\
\hline Patient had not yet initiated treatment & $0.78(0.69,0.88)$ & $<0.001^{*}$ & $0.86(0.76,0.97)$ & $0.013^{*}$ \\
\hline $2 \mathrm{~L}$ & $1.31(1.17,1.47)$ & $<0.001^{*}$ & $1.31(1.17,1.47)$ & $<0.001^{*}$ \\
\hline $3 \mathrm{~L}$ & $1.92(1.66,2.22)$ & $<0.001^{*}$ & $1.92(1.66,2.23)$ & $<0.001^{*}$ \\
\hline $4 \mathrm{~L}+$ & $3.07(2.62,3.60)$ & $<0.001^{*}$ & $3.19(2.71,3.76)$ & $<0.001^{*}$ \\
\hline \multicolumn{5}{|l|}{ Baseline characteristics (fixed covariates) } \\
\hline Age (per year increase) & $1.03(1.02,1.03)$ & $<0.001^{*}$ & $1.03(1.03,1.04)$ & $<0.001^{*}$ \\
\hline Time from initial PC diagnosis to mCRPC (per year increase) & $0.99(0.98,0.99)$ & $<0.001^{*}$ & $0.98(0.97,0.99)$ & $0.003^{*}$ \\
\hline \multicolumn{5}{|l|}{ Pre- mCRPC clinical states (ref: first mPC, then mCRPC) } \\
\hline First nmCRPC, then mCRPC & $0.88(0.80,0.97)$ & $0.009^{*}$ & $0.99(0.89,1.11)$ & 0.923 \\
\hline Metastatic and CR diagnosed at the same time pre-mCRPC & $0.70(0.48,1.04)$ & 0.075 & $0.84(0.57,1.25)$ & 0.401 \\
\hline Stage IV vs. stages I-III at initial PC diagnosis & $1.31(1.14,1.51)$ & $<0.001^{*}$ & $0.98(0.82,1.16)$ & 0.784 \\
\hline $\begin{array}{l}\text { High-risk Gleason at initial PC diagnosis } \\
(\text { score 9-10) vs. lower risk Gleason }(\text { score } \leq 8)\end{array}$ & $1.28(1.16,1.40)$ & $<0.001^{*}$ & $1.20(1.09,1.33)$ & $<0.001^{*}$ \\
\hline ECOG performance status ${ }^{\mathrm{a}}$ at $\mathrm{mCRPC}$ diagnosis $\geq 2$ vs. $0-1$ & $2.12(1.80,2.49)$ & $<0.001^{*}$ & $1.68(1.42,1.99)$ & $<0.001^{*}$ \\
\hline \multicolumn{5}{|l|}{ PSA laboratory test at mCRPC diagnosis (ref: $<4 \mathrm{ng} / \mathrm{mL}$ ) } \\
\hline $4-9$ & $1.03(0.87,1.22)$ & 0.726 & $1.00(0.84,1.18)$ & 0.956 \\
\hline $10-49$ & $1.42(1.23,1.64)$ & $<0.001^{*}$ & $1.28(1.10,1.48)$ & $0.001^{*}$ \\
\hline$\geq 50$ & $2.20(1.91,2.54)$ & $<0.001^{*}$ & $1.71(1.47,1.98)$ & $<0.001^{*}$ \\
\hline \multicolumn{5}{|l|}{ Other laboratory tests at mCRPC diagnosis } \\
\hline \multicolumn{5}{|l|}{ Hemoglobin level (ref: $\geq 14 \mathrm{~g} / \mathrm{dL})$} \\
\hline$<10$ & $3.90(3.14,4.84)$ & $<0.001^{*}$ & $1.92(1.53,2.40)$ & $<0.001^{*}$ \\
\hline $10-13$ & $1.57(1.31,1.87)$ & $<0.001^{*}$ & $1.20(1.01,1.44)$ & $0.043^{*}$ \\
\hline \multicolumn{5}{|l|}{ Serum alkaline phosphatase (ref: 44-146 U/L) } \\
\hline$<44$ & $0.78(0.55,1.12)$ & 0.182 & $0.87(0.61,1.25)$ & 0.451 \\
\hline$\geq 147$ & $2.72(2.42,3.06)$ & $<0.001^{*}$ & $1.98(1.75,2.24)$ & $<0.001^{*}$ \\
\hline Serum albumin $<34$ vs. $\geq 34 \mathrm{~g} / \mathrm{L}$ & $2.76(2.27,3.36)$ & $<0.001^{*}$ & $1.77(1.44,2.17)$ & $<0.001^{*}$ \\
\hline \multicolumn{5}{|l|}{ Lactose dehydrogenase (ref: 105-332 U/L) } \\
\hline$<105$ & $0.29(0.04,2.06)$ & 0.216 & $0.60(0.08,4.32)$ & 0.615 \\
\hline$\geq 333$ & $2.23(1.68,2.96)$ & $<0.001^{*}$ & $1.42(1.06,1.89)$ & $0.018^{*}$ \\
\hline Opioid use pre-mCRPC diagnosis & $1.43(1.28,1.61)$ & $<0.001^{*}$ & $1.36(1.20,1.54)$ & $<0.001^{*}$ \\
\hline
\end{tabular}


Table 3 continued

\begin{tabular}{|c|c|c|c|c|}
\hline & \multirow{2}{*}{\multicolumn{2}{|c|}{$\begin{array}{l}\text { Univariate regression analysis } \\
\text { Overall survival (all patients, } \\
n=4436 \text { ) }\end{array}$}} & \multirow{2}{*}{\multicolumn{2}{|c|}{$\begin{array}{l}\text { Multivariate regression analysis } \\
\text { Overall survival (all patients, } \\
n=4436 \text { ) }\end{array}$}} \\
\hline & & & & \\
\hline & HR (95\% CI) & $P$ value & HR $(95 \% C I)$ & $P$ value \\
\hline \multicolumn{5}{|c|}{ Type of primary treatment at initial PC diagnosis } \\
\hline Prostatectomy/surgery & $0.71(0.64,0.79)$ & $<0.001^{*}$ & $0.91(0.79,1.05)$ & 0.197 \\
\hline Radiation & $0.94(0.85,1.04)$ & 0.213 & $0.97(0.85,1.11)$ & 0.644 \\
\hline \multicolumn{5}{|c|}{$\begin{array}{l}C I \text { confidence interval, } C R \text { castration resistance, } E C O G \text { Eastern Cooperative Oncology Group, } H R \text { hazard ratio, } m C R P C \text { metastatic } \\
\text { castration-resistant prostate cancer, nmCRPC non-metastatic castration-resistant prostate cancer, } P C \text { prostate cancer, } P S A \text { prostate- } \\
\text { specific antigen, } A D T \text { androgen-deprivation therapy }\end{array}$} \\
\hline \multicolumn{5}{|c|}{$\begin{array}{l}\text { ECOG performance status: (0) fully active, able to carry on all pre-disease performance without restriction; (1) restricted in physically } \\
\text { strenuous activity but ambulatory and able to carry out work of a light or sedentary nature, e.g., light house work, office work; ( } 2 \text { ) } \\
\text { ambulatory and capable of all self-care but unable to carry out any work activities; up and about more than } 50 \% \text { of waking hours; ( } 3 \text { ) } \\
\text { capable of only limited self-care; confined to bed or chair more than } 50 \% \text { of waking hours; (4) completely disabled; cannot carry on any } \\
\text { self-care; totally confined to bed or chair; not documented (ECOG values of } 5 \text { are dropped) }\end{array}$} \\
\hline
\end{tabular}

treatments that prolong survival among patients with mCRPC.

The present study described treatment across the continuum of the patients' PC disease. PremCRPC diagnosis, chemotherapy was more common in metastatic states (i.e., mHSPC) than non-metastatic states (i.e., nmHSPC and nmCRPC), which is consistent with the NCCN Guidelines ${ }^{\circledR}$ for recommended treatments in the pre-mCRPC setting during the study period and thereafter [16, 27-31]. As of 2018, the FDA has expanded its approval of NHA to the premCRPC setting, including the use of abiraterone and enzalutamide for mHSPC [32-34] and apalutamide and enzalutamide for nmCRPC [33, 35]. Consistent with this, about one in five patients in the present study received NHA in the pre-mCRPC setting overall, ranging from $<5 \%$ in $\mathrm{mHSPC}$ clinical states to $34.1 \%$ in the nmCRPC clinical state. Given these high rates observed over the study period spanning from 2013 to 2019 , it is also possible that NHA were being used off-label prior to their FDA approval for the pre-mCRPC setting. The emerging use of NHA in the pre-mCRPC setting may limit the benefits of NHA for the treatment of mCRPC, primarily due to the risk of acquired resistance and cross-resistance associated with repeated NHA use $[36,37]$. However, this is less likely to be a concern among patients harboring HRR gene alterations, since they may benefit from a wider range of novel treatment options post-NHA (i.e., PARPi) compared to patients without HRR mutations.

Post-mCRPC diagnosis, the most common regimens by class were NHA, chemotherapy (i.e., docetaxel), and Sip-T, which is generally consistent with NCCN Guidelines for recommended treatments in the post-mCRPC setting during the study period and thereafter $[16,27-31,38]$. In the present study, the predominant treatment class in $1 \mathrm{~L}$ and $2 \mathrm{~L}$ was NHA (e.g., abiraterone and enzalutamide), followed by chemotherapy (i.e., docetaxel). This pattern appears more consistent with later NCCN Guidelines (2017 onward) that include the use of NHA as category 1 recommended treatments irrespective of prior docetaxel therapy $[16,28-31]$, whereas earlier guidelines primarily recommended NHA as treatment options for patients in the post-docetaxel setting or for those who are not candidates for docetaxel $[27,38]$. Further, the present findings align with those of prior real-world studies by George et al. 
[10] and Higano et al. [11] that used the same data source to assess patients during an earlier time period (2013-2017). Whereas these two prior studies did not describe treatment patterns beyond 3L $[10,11]$, the present study shows an increasing reliance on chemotherapy agents in 3L and beyond. Specifically, docetaxel was the most common regimen in $3 \mathrm{~L}$, whereas cabazitaxel became the agent of choice beyond 3L.

In the present study, approximately $50 \%$ of patients did not receive a subsequent LOT after $1 \mathrm{~L}$, which is similar to the results of prior retrospective studies based on EHR data [10, 11]. Among patients with multiple LOTs, the most common LOT sequences post-mCRPC diagnosis were NHA $\rightarrow$ NHA (in patients with $\geq 2$ LOTs) and NHA $\rightarrow$ NHA $\rightarrow$ chemotherapy (in patients with $\geq 3$ LOTs), which reflects the increasingly common use of consecutive NHA in real-world settings $[10,11]$. Interestingly, the duration of 1L NHA was longer among patients treated with NHA $\rightarrow$ NHA than patients treated with NHA $\rightarrow$ chemotherapy. These findings suggest that physicians may be seeking to maximize the benefits of NHA treatment, specifically among patients who responded to initial NHA therapy. However, given the increasingly common use of NHA in the pre-mCRPC setting, these postmCRPC treatment patterns may change in the future.

The median duration of treatment in the present study was typically $<6$ months (e.g., median duration of $1 \mathrm{~L}$ : ranging from 2.8 months with chemotherapy to 5.1 months with NHA), which is consistent with previous findings reported by George et al. [10]; in particular, George et al. observed a median time on treatment of 5.4 months for IL abiraterone and 5.8 months for $1 \mathrm{~L}$ enzalutamide, which further shortened with each successive LOT [10]. These median durations of NHA treatment are shorter than those observed in pivotal RCTs of enzalutamide and abiraterone, which ranged between 8.3 and 16.6 months for enzalutamide and between 8.0 and 13.8 months for abiraterone [39-42]. Several factors may explain the shorter duration of NHA treatment in the current study compared with the RCTs. First, a relatively high proportion of patients included in this realworld study used NHA in pre-mCRPC settings.
As more patients receive NHA in the earlier settings, the treatment patterns and subsequent time on treatment will likely evolve. Second, longer treatment durations in RCTs may also reflect the inclusion of patients with less severe disease compared to real-world populations $[43,44]$. Consistent with this, some RCTs of the safety and efficacy of NHA had selected patients that were treatment-naïve and asymptomatic or minimally symptomatic with lower ECOG performance status (e.g., scores $\leq 1$ ) $[39,42]$, while other RTCs had excluded patients with serious nonmalignant comorbidities [41]. Further, Gleason scores tended to be lower in the RCTs compared to the present study population (i.e., Gleason scores $\geq 8$ : $\sim 50$ vs. $66 \%$ of patients) $[40,41]$. Finally, patients in RCTs generally show better adherence, persistence, and compliance to therapy $[43,44]$, which may have also contributed to the longer duration of NHA treatment compared to real-world studies. Future studies should further elucidate the shorter duration of NHA treatment in realworld studies compared with RCTs.

The present study builds upon prior research by assessing treatment duration by therapeutic class rather than at the agent level, and by accounting for the impact of LOT sequencing on outcomes. In the analysis of treatment durations across specific LOT sequences, the present study observed considerable variation in time on treatment across patients, with $1 \mathrm{~L}$ NHA showing higher individual variability compared to 1L chemotherapy (IQR for time on treatment: $2.4-10.6$ vs. $1.4-4.0$ months, respectively). The time on $1 \mathrm{~L}$ and $2 \mathrm{~L}$ therapy combined was longer for NHA $\rightarrow$ NHA compared to the NHA $\rightarrow$ chemotherapy, although the time on treatment within LOT sequences tended to become shorter overall post-1L. While censoring may have been a concern in the present study, we note that treatment durations were short across all LOTs, including those that were observed to completion.

To our knowledge, the present study is the only analysis of the impact of LOTs on OS among mCRPC patients that accounts for changes in LOTs as a time-dependent covariate. This type of analysis clearly demonstrates that patients who moved more rapidly through 
multiple LOTs had a higher hazard of death at a given time point post-mCRPC diagnosis compared to those who had fewer LOTs at that time point. Prior studies have mainly focused of the impact of specific treatment sequences on OS among patients with MCRPC and were subject to several limitations [45-48]. First, they primarily focused on patients who had been treated with NHA and cabazitaxel in different sequences following progression on docetaxel, a treatment sequence that is no longer representative of current mCRPC treatment approaches [45-49]. The present study in turn captures a contemporary sample of patients with higher utilization of NHA. Second, studies comparing specific regimen sequences may be subject to confounding due to unmeasured clinical factors that influence regimen choices. An analysis of the impact of LOT number on OS, irrespective of the regimens used in each LOT, avoids this type of bias. Finally, prior studies did not account for censorship and the time-dependent nature of LOT changes post-mCRPC diagnosis. While one prior study did use a Cox regression model to assess the impact of LOT on OS, the LOTs were included as fixed variables in the model [47].

One of the main challenges in the treatment of mCRPC is the molecular heterogeneity of the disease [50, 51]. With the approval of PARPi, more treatment options have become available in 2020 for patients with HRR-mutated mCRPC who have progressed on conventional therapy $[12,13,52]$. Although consecutive NHA are currently the most common treatment sequence in $1 \mathrm{~L}$ and $2 \mathrm{~L}$, the PROfound trial has shown a clear survival advantage with NHA $\rightarrow$ olaparib among patients with alterations in at least 1 of 14 HRR genes, including $B R C A 1 / 2$, following progression on NHA [12]. Moreover, patients with $B R C A 1 / 2$ gene alterations may benefit from rucaparib following progression on androgen receptor-directed therapy and taxane chemotherapy [53]. The availability of novel targeted therapies such as PARPi now provide a rationale for routine testing of appropriate patients for HRR mutations per clinical guidelines [31].

The present study had certain limitations. First, treatment selection in $2 \mathrm{~L}$ and later lines is typically based on pre-existing patient characteristics (e.g., fitness level, response to prior LOTs), which may have influenced treatment outcomes; further, certain clinically important patient characteristics (i.e., comorbidities) were underreported in EHR data and, therefore, could not be adjusted for in the multivariate regression model for OS. Second, some patients may have received care outside the Flatiron Health network during the study period; in particular, treatments early in the course of PC disease may not have been fully captured for patients who had received their initial PC diagnosis outside the Flatiron Health network. Third, for most patients who did not die during the study period, censoring due to end of data availability underestimated the time on treatment associated with the patient's last observed LOT. Fourth, LOTs were inferred from patterns of treatment administrations/orders recorded in the EHR data, and therefore occasional misclassification may have occurred. Fifth, diseasespecific mortality data was not available in the database. Finally, the data used in this study are mostly drawn from community oncology practices and may not be representative of academic research centers.

\section{CONCLUSION}

In this real-world study in the US, consecutive LOTs with NHA remained the preferred treatment option. Despite a large number of LOT sequences beyond $1 \mathrm{~L}$, most patients died after one or two LOTs, highlighting a significant unmet need. The therapeutic landscape for mCRPC is continuing to evolve and treatments aimed at addressing the molecularly heterogeneity of the disease have emerged. In particular, recently approved PARPi may improve clinical outcomes and prolong survival among eligible patients. Updated NCCN Guidelines recommending genomic testing for HRR gene alterations among appropriate patients [31], combined with growing physician awareness of the importance of genetic testing in guiding treatment choices, have the potential to further transform the treatment patterns in mCRPC. 


\section{ACKNOWLEDGEMENTS}

Funding. This work and the journal's Rapid Service and Open Access Fees were funded by Merck Sharp \& Dohme Corp., a subsidiary of Merck \& Co., Inc., Kenilworth, NJ, USA and AstraZeneca UK Limited.

Medical Writing, Editorial, and Other Assistance. Medical writing assistance was provided by Mona Lisa Chanda, PhD, an employee of Analysis Group, Inc. Support for this assistance was provided by Merck Sharp \& Dohme Corp. and AstraZeneca UK Limited. Feedback on the study and manuscript were provided by Alicia Gayle, Suvina Amin, and Sarah Payne, who are employees of AstraZeneca.

Authorship. All named authors meet the International Committee of Medical Journal Editors (ICMJE) criteria for authorship for this article, take responsibility for the integrity of the work as a whole, and have given their approval for this version to be published.

Authorship Contributions. All authors contributed to the study concept/design and interpretation of data. Sameer R. Ghate acquired the data. Malena Mahendran, Dominique Lejeune, Louise H.Yu, Raluca Ionescu-Ittu, François Laliberté, and Mei Sheng Duh performed the analyses. All authors drafted and critically revised the present manuscript for important intellectual content. All authors approve the version to be published and agree to be accountable for all aspects of the work in ensuring that questions related to the accuracy or integrity of any part of the work are appropriately investigated and resolved.

Disclosures. Neal D. Shore has received compensation as a consultant and researcher from Amgen, Astellas, AstraZeneca, Bayer, BMS, Boston Scientific, Dendreon, Ferring, Fergene, Janssen, MDx Health, Merck, Myovant, Nymox, Pfizer, Sanofi-Genzyme, and Tolmar. Raluca Ionescu-Ittu, François Laliberté, Malena Mahendran, Dominique Lejeune, Louise H.Yu, and Mei Sheng Duh are employees of Analysis
Group, Inc., a company that has received funding from Merck and has provided paid consulting services to various clients in the biomedical arena. Lingfeng Yang, Joseph Burgents, and Sameer R. Ghate are employees of Merck Sharp \& Dohme Corp., a subsidiary of Merck \& Co., Inc., Kenilworth, NJ, USA, as well as shareholders/stockholders in Merck \& Co., Inc., Kenilworth, NJ, USA.

Compliance with Ethics Guidelines. All database records were statistically de-identified and certified as fully compliant with US patient confidentiality requirements outlined in the Health Insurance Portability and Accountability Act of 1996. Because this study relied exclusively on de-identified patient records and did not involve the collection, use, or dissemination of individually-identifiable data, institutional review board approval was not necessary.

Data Availability. The datasets generated and/or analyzed during the current study are not publicly available per the terms of the data use agreement between the study investigators and the data vendor.

Open Access. This article is licensed under a Creative Commons Attribution-NonCommercial 4.0 International License, which permits any non-commercial use, sharing, adaptation, distribution and reproduction in any medium or format, as long as you give appropriate credit to the original author(s) and the source, provide a link to the Creative Commons licence, and indicate if changes were made. The images or other third party material in this article are included in the article's Creative Commons licence, unless indicated otherwise in a credit line to the material. If material is not included in the article's Creative Commons licence and your intended use is not permitted by statutory regulation or exceeds the permitted use, you will need to obtain permission directly from the copyright holder. To view a copy of this licence, visit http://creativecommons.org/licenses/by$\mathrm{nc} / 4.0 /$. 


\section{REFERENCES}

1. https://seer.cancer.gov/statfacts/html/prost.html. Accessed Sep 24, 2020.

2. Centers for disease control and prevention: prostate cancer statistics. 2020. https://www.cdc.gov/ cancer/prostate/statistics/index.htm. Accessed Jul $13,2020$.

3. Hotte SJ, Saad F. Current management of castrateresistant prostate cancer. Curr Oncol. 2010;17(Suppl 2):S72-9. https://doi.org/10.3747/ co.v17i0.718.

4. Kirby M, Hirst C, Crawford ED. Characterising the castration-resistant prostate cancer population: a systematic review. Int J Clin Pract. 2011;65(11): 1180-92. https://doi.org/10.1111/j.1742-1241. 2011.02799.x

5. Moreira DM, Howard LE, Sourbeer KN, Amarasekara HS, Chow LC, Cockrell DC, et al. Predicting time from metastasis to overall survival in castration-resistant prostate cancer: results from SEARCH. Clin Genitourin Cancer. 2017;15(1):60-6. https://doi. org/10.1016/j.clgc.2016.08.018 (e2).

6. Mateo J, Boysen G, Barbieri CE, Bryant HE, Castro E, Nelson PS, et al. DNA repair in prostate cancer: biology and clinical implications. Eur Urol. 2017;71(3):417-25. https://doi.org/10.1016/j. eururo.2016.08.037.

7. Corfield J, Crozier J, Joshua AM, Bolton D, Lawrentschuk $\mathrm{N}$. Understanding the role of new systemic agents in the treatment of prostate cancer. BJU Int. 2016;118(Suppl 3):8-13. https://doi.org/10. 1111/bju.13633.

8. Wade CA, Kyprianou N. Profiling prostate cancer therapeutic resistance. Int J Mol Sci. 2018. https:// doi.org/10.3390/ijms19030904.

9. Halwani AS, Rasmussen KM, Patil V, Li CC, Yong $\mathrm{CM}$, Burningham $Z$, et al. Real-world practice patterns in veterans with metastatic castration-resistant prostate cancer. Urol Oncol. 2020;38(1):1ee10. https://doi.org/10.1016/j.urolonc.2019.09. 027.

10. George DJ, Sartor O, Miller K, Saad F, Tombal B, Kalinovsky J, et al. Treatment patterns and outcomes in patients with metastatic castration-resistant prostate cancer in a real-world clinical practice setting in the United States. Clin Genitourin Cancer. 2020. https://doi.org/10.1016/j.clgc.2019.12. 019.

11. Higano CS, Sternberg CN, Saad F, Tombal BF, Miller $\mathrm{K}$, Kalinovsky J, et al. Treatment patterns and outcomes for metastatic castration-resistant prostate cancer (mCRPC) in a real-world setting: A retrospective study of greater than 2500 patients. Am Soc Clin Oncol. 2019;37(7_suppl):256.

12. de Bono J, Mateo J, Fizazi K, Saad F, Shore N, Sandhu S, et al. Olaparib for metastatic castrationresistant prostate cancer. $\mathrm{N}$ Engl J Med. 2020;382(22):2091-102. https://doi.org/10.1056/ NEJMoa1911440.

13. United States Food and Drug Administration: RUBRACA ${ }^{\circledR}$ (rucaparib)—accelerated approval. 2020,https://www.accessdata.fda.gov/drugsatfda_ docs/appletter/2020/209115Orig1s004ltr.pdf. Accessed Jun 24, 2020.

14. United States Food and Drug Administration: FDA grants accelerated approval to rucaparib for BRCAmutated metastatic castration-resistant prostate cancer. 2020. https://www.fda.gov/drugs/fdagrants-accelerated-approval-rucaparib-brcamutated-metastatic-castration-resistant-prostate. Accessed Sep 25, 2020.

15. Abida W, Patnaik A, Campbell D, Shapiro J, Bryce $\mathrm{AH}, \mathrm{McDermott} \mathrm{R}$, et al. Rucaparib in men with metastatic castration-resistant prostate cancer harboring a BRCA1 or BRCA2 gene alteration. J Clin Oncol. 2020;38(32):3763.

16. Referenced with permission from the NCCN Clinical Practice Guidelines in Oncology (NCCN Guidelines ${ }^{\circledR}$ ) for Prostate Cancer-Version 2.2020. (C) National Comprehensive Cancer Network, Inc. 2020. All rights reserved. Accessed [June 24, 2020]. To view the most recent and complete version of the guideline, go online to NCCN.org. Accessed.

17. Moses KA, Wallace $\mathrm{K}$, Landsteiner A, Bunner $\mathrm{S}$, Engel-Nitz N, Lal LS, et al. Real-world evidence of treatment patterns and pharmacy costs among patients with metastatic castration-resistant prostate cancer (mCRPC) in a managed care population in the United States. J Clin Oncol. 2020. https://doi. org/10.1200/JCO.2020.38.15_suppl.e19319.

18. Wen L, Yao J, Valderrama A. Evaluation of treatment patterns and costs in patients with prostate cancer and bone metastases. J Manag Care Spec Pharm. 2019;25(3-b Suppl):S1-11. https://doi.org/ 10.18553/jmcp.2019.25.3-b.s1.

19. Sonpavde G, Huang A, Wang L, Baser O, Miao R. Taxane chemotherapy vs antiandrogen agents as first-line therapy for metastatic castration-resistant prostate cancer. BJU Int. 2018;121(6):871-9. https://doi.org/10.1111/bju.14152.

20. George DJ, Sternberg CN, Sartor AO, Saad F, Tombal $\mathrm{BF}$, Miller K, et al. Clinical outcome with concurrent or layered treatment with radium-223 and 
abiraterone: A retrospective study of real-world experience with patients (pts) with metastatic castration-resistant prostate cancer (mCRPC). Am Soc Clin Oncol. 2019;37(7_suppl):253.

21. Flatiron Health Database. 2020. https://flatiron. com/real-world-evidence/. Accessed Jun 18, 2020.

22. Ma X, Long L, Moon S, Adamson BJS, Baxi SS. Comparison of population characteristics in realworld clinical oncology databases in the US: Flatiron Health, SEER, and NPCR. medrRxiv. 2020;105: 912.

23. Scher HI, Solo K, Valant J, Todd MB, Mehra M. Prevalence of prostate cancer clinical states and mortality in the United States: estimates using a dynamic progression model. PLOS ONE. 2015;10(10): e0139440. https://doi.org/10.1371/ journal.pone.0139440.

24. Scher HI, Heller G. Clinical states in prostate cancer: toward a dynamic model of disease progression. Urology. 2000;55(3):323-7. https://doi.org/10. 1016/s0090-4295(99)00471-9.

25. Lam J, Yang C, Kaiser C, Wong W. Real-world treatment patterns and care pathways in metastatic castration resistant prostate cancer. Value Health. 2018;21:S41.

26. Curtis MD, Griffith SD, Tucker M, Taylor MD, Capra WB, Carrigan G, et al. Development and validation of a high-quality composite real-world mortality endpoint. Health Serv Res. 2018;53(6):4460-76. https://doi.org/10.1111/1475-6773.12872.

27. Referenced with permission from the NCCN Clinical Practice Guidelines in Oncology (NCCN Guidelines () for Prostate Cancer-Version 3.2016. (C) National Comprehensive Cancer Network, Inc. 2016. All rights reserved. Accessed [June 24, 2020]. To view the most recent and complete version of the guideline, go online to NCCN.org. Accessed.

28. Referenced with permission from the NCCN Clinical Practice Guidelines in Oncology (NCCN Guidelines $\left.{ }^{\circledR}\right)$ for Prostate Cancer-Version 2.2017. ( $)$ National Comprehensive Cancer Network, Inc. 2017. All rights reserved. Accessed [Jun 24, 2020]. To view the most recent and complete version of the guideline, go online to NCCN.org. Accessed.

29. Referenced with permission from the NCCN Clinical Practice Guidelines in Oncology (NCCN Guidelines $\left.{ }^{\circledR}\right)$ for Prostate Cancer-Version 4.2018. (C) National Comprehensive Cancer Network, Inc. 2018. All rights reserved. Accessed [Jun 24, 2020]. To view the most recent and complete version of the guideline, go online to NCCN.org. Accessed.
30. Referenced with permission from the NCCN Clinical Practice Guidelines in Oncology (NCCN Guidelines $\left.{ }^{\circledR}\right)$ for Prostate Cancer-Version 2.2019. (ㄷ National Comprehensive Cancer Network, Inc. 2019. All rights reserved. Accessed [Jun 24, 2020]. To view the most recent and complete version of the guideline, go online to NCCN.org. Accessed.

31. Referenced with permission from the NCCN Clinical Practice Guidelines in Oncology (NCCN Guidelines $\left.{ }^{\circledR}\right)$ for Prostate Cancer-Version 2.2021. () National Comprehensive Cancer Network, Inc. 2021. All rights reserved. Accessed [Feb 18, 2021]. To view the most recent and complete version of the guideline, go online to NCCN.org. NCCN makes no warranties of any kind whatsoever regarding their content, use or application and disclaims any responsibility for their application or use in any way.: Accessed.

32. Janssen Pharmaceutical Companies: ZYTIGA ${ }^{\circledR}$ (abiraterone acetate) Plus Prednisone Approved for Treatment of Earlier Form of Metastatic Prostate Cancer. 2018. https://www.prnewswire.com/newsreleases/zytiga-abiraterone-acetate-plusprednisone-approved-for-treatment-of-earlier-formof-metastatic-prostate-cancer-300595759.html. Accessed Jun 24, 2020.

33. XTANDI® (enzalutamide) Capsules, for Oral Use [Prescribing Information]: United States Food and Drug Administration; 2019. https://www. accessdata.fda.gov/drugsatfda_docs/label/2019/ 203415s015lbl.pdf. Accessed Jun, 242020.

34. ZYTIGA ${ }^{\circledR}$ (abiraterone acetate) Tablets, for Oral Use [Prescribing Information]: United States Food and Drug Administration; 2020. https://www. accessdata.fda.gov/drugsatfda_docs/label/2020/ 202379s031s033lbl.pdf. Accessed Jun, 242020.

35. ERLEADA ${ }^{\circledR}$ (apalutamide) Tablets [Prescribing Information]: United States Food and Drug Administration; 2018. https://www.accessdata.fda. gov/drugsatfda_docs/label/2018/210951s000lbl. pdf. Accessed Jun 24, 2020.

36. Lorente D, Fizazi K, Sweeney C, de Bono JS. Optimal treatment sequence for metastatic castration-resistant prostate cancer. Eur Urol Focus. 2016;2(5): 488-98. https://doi.org/10.1016/j.euf.2016.10.008.

37. Wallis CJD, Klaassen Z. What is changing in advanced prostate cancer? Everyday Urol. $2021 ; 5(2)$.

38. Referenced with permission from the NCCN Clinical Practice Guidelines in Oncology (NCCN Guidelines $\left.{ }^{\circledR}\right)$ for Prostate Cancer-Version 2.2014. (C) National Comprehensive Cancer Network, Inc. 2014. All rights reserved. Accessed [Jun, 24, 2020]. 
To view the most recent and complete version of the guideline, go online to NCCN.org. Accessed.

39. Ryan CJ, Smith MR, Fizazi K, Saad F, Mulders PF, Sternberg $\mathrm{CN}$, et al. Abiraterone acetate plus prednisone versus placebo plus prednisone in chemotherapy-naive men with metastatic castration-resistant prostate cancer (COU-AA-302): final overall survival analysis of a randomised, doubleblind, placebo-controlled phase 3 study. Lancet Oncol. 2015;16(2):152-60. https://doi.org/10.1016/ S1470-2045(14)71205-7.

40. Scher HI, Fizazi K, Saad F, Taplin ME, Sternberg CN, Miller K, et al. Increased survival with enzalutamide in prostate cancer after chemotherapy. N Engl J Med. 2012;367(13):1187-97. https://doi.org/10. 1056/NEJMoa1207506.

41. de Bono JS, Logothetis CJ, Molina A, Fizazi K, North $\mathrm{S}, \mathrm{Chu} \mathrm{L}$, et al. Abiraterone and increased survival in metastatic prostate cancer. $\mathrm{N}$ Engl J Med. 2011;364(21):1995-2005.

42. Beer TM, Armstrong AJ, Rathkopf DE, Loriot $\mathrm{Y}$, Sternberg CN, Higano CS, et al. Enzalutamide in metastatic prostate cancer before chemotherapy. N Engl J Med. 2014;371(5):424-33. https://doi.org/ 10.1056/NEJMoa1405095.

43. Roberts MH, Ferguson GT. Real-world evidence: bridging gaps in evidence to guide payer decisions. Pharmacoecon Open. 2021;5(1):3-11. https://doi. org/10.1007/s41669-020-00221-y.

44. Blumenthal DM, Yu-Isenberg $\mathrm{K}$, Yee J, Jena AB. Real-world evidence complements randomized controlled trials in clinical decision making. Health Affairs Blog. 2017.

45. Angelergues A, Efstathiou E, Gyftaki R, Wysocki PJ, Lainez N, Gonzalez I, et al. Results of the FLAC European database of metastatic castration-resistant prostate cancer patients treated with docetaxel, cabazitaxel, and androgen receptor-targeted agents. Clin Genitourin Cancer. 2018;16(4):e777-84. https://doi.org/10.1016/j.clgc.2018.02.016.

46. Maines F, Caffo O, Veccia A, Trentin C, Tortora G, Galligioni E, et al. Sequencing new agents after docetaxel in patients with metastatic castration-resistant prostate cancer. Crit Rev Oncol Hematol. 2015;96(3):498-506. https://doi.org/10.1016/j. critrevonc.2015.07.013.
47. Sonpavde G, Bhor M, Hennessy D, Bhowmik D, Shen L, Nicacio L, et al. Sequencing of cabazitaxel and abiraterone acetate after docetaxel in metastatic castration-resistant prostate cancer: treatment patterns and clinical outcomes in multicenter community-based US oncology practices. Clin Genitourin Cancer. 2015;13(4):309-18. https://doi. org/10.1016/j.clgc.2014.12.019.

48. Caffo O, Wissing M, Bianchini D, Bergman A, Thomsen FB, Schmid S, et al. Survival outcomes from a cumulative analysis of worldwide observational studies on sequential use of new agents in metastatic castration-resistant prostate cancer. Clin Genitourin Cancer. 2020;18(1):69-76. https://doi. org/10.1016/j.clgc.2019.09.010 (e4).

49. Caffo O, Bria E, Giorgi UD, Tucci M, Galli L, Fratino $\mathrm{L}$, et al. Outcomes of metastatic castration-resistant prostate cancer (mCRPC) patients (pts) treated with different new agents (NAs) sequence in post-docetaxel (DOC) setting: final analysis from a multicenter Italian study. J Clin Oncol. 2017;35(15_suppl):5030. https://doi.org/10.1200/ JCO.2017.35.15_suppl.5030.

50. Modena A, Iacovelli R, Scarpa A, Brunelli M, Ciccarese C, Fantinel E, et al. Investigating BRCA mutations: a breakthrough in precision medicine of castration-resistant prostate cancer. Target Oncol. 2016;11(5):569-77. https://doi.org/10.1007/ s11523-016-0450-9.

51. $\mathrm{Ku}$ SY, Gleave ME, Beltran H. Towards precision oncology in advanced prostate cancer. Nat Rev Urol. 2019;16(11):645-54. https://doi.org/10.1038/ s41585-019-0237-8.

52. Smith MR, Sandhu SK, Kelly WK, Scher HI, Efstathiou E, Lara P, et al. Phase II study of niraparib in patients with metastatic castration-resistant prostate cancer (mCRPC) and biallelic DNA-repair gene defects (DRD): preliminary results of GALAHAD. Am Soc Clin Oncol. 2019;30:v884-5.

53. RUBRACA ${ }^{\circledR}$ (rucaparib) Tablets, for Oral Use [Prescribing Information]: United States Food and Drug Administration; 2020. https://www.accessdata.fda. gov/drugsatfda_docs/label/2020/209115s004lbl.pdf Accessed Jun 24, 2020. 